EESTI NSV TEADUSTE AKADEEMIA TOIMETISED. 28. KOIDE

FOOSIKA * MATEMAATIKA. 1979, NR. I

ИЗВЕСТИЯ АКАДЕМИИ НАУК ЭСТОНСКОП ССР. ТОМ 28 ФИЗНКА * MATЕМАТИКА. 1979, № 1

удК $62-501-72$

Юлле КОТТА

\title{
ИДЕНТИФИКАЦИЯ ЛИНЕИНОГО ОБЪЕКТА В ЗАМКНУТОЙ СИСТЕМЕ
}

(Представлена Н. Алумяэ)

\section{1. Постановка задачи}

При построении моделей часто приходится прибегать к идентификации объектов в процессе их нормального функционирования. Основной задачей при этом является получение алгоритмов идентификации, предназначенных для оценивания параметров объекта в системе с обратной связью.

Известно [ $\left.{ }^{1}\right]$ два типа методов идентификации прямой цепи замкнутой системы - косвенные и прямые. С помощью косвенных сначала оценивают характеристики всей замкнутой системы, а затем вычисляют характеристики объекта с привлечением информации о параметрах регулятора. Эти методы предполагают точное знание характеристики обратной связи. Прямые методы лишены указанного недостатка, с их помощыю данные «вход-выход» обрабатывают так же, как и данные в эксперименте с разомкнутым контуром. Однако здесь возникает другая проблема. В основе почти всех доказательств результатов о поведении оценок параметров объекта лежит допущение, что его вход статистически независим от выхода, т. е. они базируются на предположении, которое не имеет силы для объектов в замкнутом контуре. Поэтому для разрешимости задачи оценивания прямыми методами важно отыскать условия, которым бы удовлетворяли как объект, так и обратная связь.

В данной работе будем искать условия состоятельности оценок параметров. Как известно [ $\left.{ }^{1}\right]$, решение этой задачи зависит от структуры модели, применяемого метода идентификации, характера системы и условий проведения эксперимента.

Рассмотрим линейный, стационарный, дискретный, стохастический объект управления с одним входом и одним выходом. Такие объекты описываются в общем виде выражениями

$$
y(t)=G\left(q^{-1}\right) u(t)+H\left(q^{-1}\right) e(t),
$$

где переменные $\{e(t)\}$ представляют собой последовательность независимых случайных величин с нулевыми средними и дисперсиями $\sigma^{2}$, $q^{-1}-$ оператор обратного сдвига, $q^{-1} u(t)=u(t-1), G\left(q^{-1}\right), H\left(q^{-1}\right)-$ рациональные функции аргумента $q^{-1}$.

Для определения модели системы функции $G\left(q^{-1}\right)$ и $H\left(q^{-1}\right)$ надо параметризовать с помощыю вектора параметров $\theta$. Модель, соответствующая определенному значению $\theta$, задается уравнением 


$$
y(t)=G_{\theta}\left(q^{-1}\right) u(t)+H_{\theta}\left(q^{-1}\right) \varepsilon(t),
$$

где $\{\varepsilon(t)\}$ - последовательность независимых случайных величин с нулевыми средними и дисперсиями $\sigma_{\varepsilon}{ }^{2}$.

Параметризация $G\left(q^{-1}\right)$ и $H\left(q^{-1}\right)$ может быть выполнена несколькими способами, обычно используют разностное уравнение

$$
A_{\theta}\left(q^{-1}\right) y(t)=B_{\theta}\left(q^{-1}\right) u(t)+C_{\theta}\left(q^{-1}\right) \varepsilon(t),
$$

где

$$
\begin{aligned}
& A_{\theta}\left(q^{-1}\right)=1+\sum_{i=1}^{n_{a}} a_{i} q^{-i}, \\
& B_{\theta}\left(q^{-1}\right)=\sum_{i=p}^{n_{b}} b_{i} q^{-i}, \\
& C_{\theta}\left(q^{-1}\right)=1+\sum_{i=1}^{n_{\mathrm{c}}} c_{i} q^{-i} .
\end{aligned}
$$

Задаче отыскания условий состоятельности оценок параметров указанной модели в настоящее время придается большое значение. Отыскивают условия в зависимости от выбранного вида полинома $C_{\theta}\left(q^{-1}\right)\left(C_{\theta}\left(q^{-1}\right)=1\left[{ }^{2}\right]\right)$, от выбранного метода идентификации (в $\left[{ }^{2,3}\right]$ - это метод наименьших квадратов, в [ [4-6] - метод максимального правдоподобия) и от заданных условий проведения эксперимента (различные сдвиги в законе обратной связи $\left[{ }^{7}\right]$, существование внешних входных сигналов $\left.\left[{ }^{1}\right]\right)$.

В данной работе предполагается, что $C_{\theta}\left(q^{-1}\right)=A_{\theta}\left(q^{-1}\right)$. Это соответствует частному случаю, когда сам объект является детерминированным, а выходы измеряются с некоррелированными во времени ошибками. Для идентификации такой модели, кроме метода максимального правдоподобия, пригоден метод собственного вектора, который (в случае нормального распределения ошибок измерений) приближенно вычисляет оценки максимального правдоподобия с помощью аппроксимации функции правдоподобия, хорошо зарекомендовавшей себя при идентификации динамики разомкнутых объектов [ $\left.{ }^{8}\right]$.

Предполагается, кроме того, что управляющее воздействие осуществляется регулятором в обратной связи по закону

$$
F\left(q^{-1}\right) u(t)=D\left(q^{-1}\right) y(t)
$$

или

$$
F\left(q^{-1}\right) u(t)=D\left(q^{-1}\right) y(t)+F\left(q^{-1}\right) \delta(t)
$$

где

$$
\begin{gathered}
F\left(q^{-1}\right)=1+\sum_{i=1}^{n_{f}} f_{i} q^{-i}, \\
D\left(q^{-1}\right)=\sum_{i=k}^{n_{a}} d_{i} q^{-i},
\end{gathered}
$$

и переменная $\delta(t)$ является внешним возмущением относительно выходного сигнала регулятора в обратной связи. Принимается, что $\delta(t)-$ некоррелированные во времени случайные величины с нулевыми средними и дисперсиями $\sigma_{\delta}^{2}-$ независимы также и от случайных величин $\varepsilon(t)$. 
Цель работы состоит в отыскании условий, при выполнении которых оценки максимального правдоподобия и оценки собственного вектора параметров объекта сходятся почти наверное (п. н.) к истинным значениям при $N \rightarrow \infty$ для систем (3), (4) и (3), (5).

\section{2. Основные предположения и обозначения}

Введем вектор параметров объекта $\theta^{\prime}=\left(\theta_{1}{ }^{\prime} \theta_{2}{ }^{\prime}\right)$, где $\theta_{1}^{\prime}=\left(a_{1} a_{2} \ldots a_{n_{a}}\right)$, $\theta_{2}^{\prime}=\left(b_{p} b_{p+1} \ldots b_{n_{b}}\right)$.

Для компактности записи уравнений системы и описания доказательств используем теплицевы матрицы размера $N \times N$ :

$$
\begin{array}{ll}
\mathbf{A}=\mathbf{I}+\sum_{i=1}^{n_{a}} a_{i} \mathbf{S}^{i}, & \mathbf{B}=\sum_{i=p}^{n_{b}} b_{i} \mathbf{S}^{i}, \\
\mathbf{F}=\mathbf{I}+\sum_{i=1}^{n_{f}} f_{i} \mathbf{S}^{i}, & \mathbf{D}=\sum_{i=k}^{n_{a}} d_{i} \mathbf{S}^{i},
\end{array}
$$

где $\mathrm{S}=\left\{s_{i j}=\delta_{i, j+1}\right\}, \mathrm{S}^{i}-i$-я степень матрицы $\mathrm{S}$, и векторы

$$
\begin{aligned}
& \mathbf{y}^{\prime}=\left[\begin{array}{llll}
y(1) & y(2) & \ldots & y(N)
\end{array}\right], \\
& \mathbf{u}^{\prime}=\left[\begin{array}{llll}
u(1) & u(2) & \ldots & u(N)
\end{array}\right], \\
& \boldsymbol{\varepsilon}^{\prime}=\left[\begin{array}{llll}
\varepsilon(1) & \varepsilon(2) & \ldots & \varepsilon(N)
\end{array}\right], \\
& {\delta^{\prime}}_{N}=\left[\begin{array}{llll}
\delta(1) & \delta(2) & \ldots & \delta(N)
\end{array}\right] .
\end{aligned}
$$

Уравнения (3)-(5) при нулевых начальных значениях перепишем в виде

$$
\begin{aligned}
& \mathbf{A} \mathbf{y}_{N}=\mathbf{B} \mathbf{u}_{N}+\mathbf{A} \varepsilon_{N}, \\
& \mathbf{F} \mathbf{u}_{N}=\mathbf{D} \mathbf{y}_{N}, \\
& \mathbf{F} \mathbf{u}_{N}=\mathbf{D} \mathbf{y}_{N}+\mathbf{F} \delta_{N} .
\end{aligned}
$$

Последняя форма записи нашла широкое применение благодаря удобству алгебраических манипуляций, т. к. матрицы иј множества $T$ (нижние треугольные теплицевы матрицы) обладают следующими свойствами $\left[{ }^{9}\right]$ :

$$
\begin{aligned}
& \mathbf{A}, \mathbf{B} \in T \Rightarrow \text { 1) } \mathbf{A}^{-1} \in T, \text { если } a_{0} \neq 0, \\
& \text { 2) } \mathbf{A} \mathbf{B}=\mathbf{B} \mathbf{A} \in T .
\end{aligned}
$$

Выпишем функции $J_{N}(\theta)$ и $J_{N}^{a}(\theta)$, которые надо минимизировать для получения оценок максимального правдоподобия и оценок собственного вектора соответственно. При больших выборках влиянием начальных условий можно пренебречь, поэтому принимаем, что в исследуемых системах начальные условия нулевые. Тогда функция правдоподобия запишется в виде

$$
\begin{gathered}
L\left(\mathbf{y}_{N} \mid \theta\right)=\text { const } \times \exp \left(-\frac{1}{2 \sigma_{\varepsilon}^{2}}\left\|\mathbf{y}_{N}-\mathbf{A}^{-1} \mathbf{B} \mathbf{u}_{N}\right\|^{2}\right)= \\
=\text { const } \times \exp \left(-\frac{1}{2 \sigma_{\varepsilon}^{2}}\left\|\mathbf{A} \mathbf{y}_{N}-\mathbf{B} \mathbf{u}_{N}\right\|_{\left(\mathbf{A A}^{\prime}\right)^{-1}}^{2}\right) .
\end{gathered}
$$


Отсюда следует, что функция, минимизируемая для получения оценок максимального правдоподобия, имеет вид

$$
J_{N}(\theta)=\frac{1}{\sigma_{\varepsilon}^{2}}\left\|\mathbf{A} \mathbf{y}_{N}-\mathbf{B} \mathbf{u}_{N}\right\|_{\left(\mathrm{AA}^{\prime}\right)^{-1}}^{2} .
$$

Если весовую матрицу $\mathbf{A A}^{\prime}$ заменить матрицей $\left(1+\theta_{1}{ }^{\prime} \theta_{1}\right) \mathbf{I}$, т. е. матрицей, которая состоит только из диагональных элементов матрицы $\mathbf{A A}^{\prime}$, то для получения оценок собственного вектора $\left[{ }^{8}\right]$ найдем минимизируемую функцию

$$
J_{N}^{a}(\theta)=\frac{1}{\sigma_{\varepsilon}^{2}\left(1+\theta^{\prime}{ }_{1} \theta_{1}\right)}\left\|\mathbf{A y}_{N}-\mathbf{B} \mathbf{u}_{N}\right\|^{2} .
$$

Пусть $\left(^{0}\right)$ - индекс истинного значения.

П ред положе н и е А. Нули полинома $P_{\theta^{\circ}}(z)=A_{\theta^{\circ}}(z) F(z)-B_{\theta^{\circ}}(z) \times$ $\times D(z)$ лежат вне единичного круга.

Предположение гарантирует устойчивость замкнутой системы и обеспечивает сходимость функций $\frac{1}{N} J_{N}(\theta)$ и $\frac{1}{N} J_{N}^{a}(\theta)$ п. н. к $E J_{N}(\theta)$ и $E J_{N}^{a}(\theta)$ соответственно.

П р едположени е Б. Полиномы $A_{\theta^{\circ}}(z) u B_{\theta^{\circ}}(z)$ не имеют обцих корней $и$ не все $b_{i}{ }^{0}=0, i=p, p+1, \ldots, n_{b}$.

Это предположение необходимо для полной управляемости объекта $\left[{ }^{10}\right]$. Если идентифицируемый объект не является полностью управляемым, то значение $\theta^{0}$ не будет единственным, т. е. множества

$$
\left\{\theta: J^{a}(\theta)=J^{a}\left(\theta^{0}\right)\right\} \quad \text { и } \quad\left\{\theta: J(\theta)=J\left(\theta^{0}\right)\right\},
$$

где

$$
J^{a}(\theta)=\lim _{N \rightarrow \infty} \frac{1}{N} J_{N}^{a}(\theta) \quad \text { и } \quad J(\theta)=\lim _{N \rightarrow \infty} \frac{1}{N} J_{N}(\theta),
$$

не будут одноточечными.

Предположен и е В. $\quad$ в замкнутой системе имеется запаздывание $p+k \geqslant 1$, реализуемое или обгектом, или обратной связью.

Предположение В1. В замкнутой системе имеется запаздывание $p+k \geqslant n_{a}+1$, реализуемое или объектом, или обратной связью, или частично обтектом и частично обратной связью.

Предположения В и В1 обеспечивают, что $J\left(\theta^{0}\right) \leqslant J(\theta)$ и $J^{a}\left(\theta^{0}\right) \leqslant J^{a}(\theta)$.

Пр ед положе н и е Д. Множество $\Theta$ допустимых параметров $\theta$ есть компактное подмножество в $R^{n_{a}+n_{o}-p+1}$, причем истинное значение вектора параметров $\theta^{0}$ является внутренней точкой множества $\Theta$.

Предположение гарантирует, что последовательность оценок $\theta_{N}$ имеет сходящуюся подпоследовательность.

\section{3. Основные результаты}

Решение поставленных задач содержится в утверждениях теорем $1-4$. Т е орем а 1. Если для системы (3), (4) справедливы условия А. Б, Д, В1, то оценки собственного вектора неизвестных параметров обтекта сходятся п. н. к истинным значениям при $N \rightarrow \infty$.

Т е о рем а 2. Если для системь (3), (4) справедливы условия А, Б, В, 
Д, то оценки максимального правдоподобия неизвестных параметров обтекта сходятся п. н. к истинным значениям при $N \rightarrow \infty$.

Т е о рем а 3. Если для системы (3), (5) справедливы условия А, Б, В1, Д, то оценки собственного вектора неизвестных параметров обтекта сходятся п. н. к истинным значениям при $N \rightarrow \infty$.

Т е о р е м а 4. Если для системь (3), (5) справедливы условия А, Б, В, Д, то оценки максимального правдоподобия неизвестных параметров обтекта сходятся $n$. н. к истинным значениям при $N \rightarrow \infty$.

В работе доказана теорема 1. Доказательства остальных теорем аналогичны,

\section{4. Доказательство теоремы 1}

Нам понадобятся следующие леммы.

Л ем а $1\left[{ }^{11}\right]$. Пусть $\mathbf{x}_{N}=\mathbf{G v}_{N} u \mathbf{z}_{N}=\mathrm{He}_{N}$, ade

$$
\begin{aligned}
\mathbf{x}^{\prime}{ }_{N}=\left[\begin{array}{llllll}
x(1) & x(2) & \ldots & x(N)
\end{array}\right], & \mathbf{z}_{N}^{\prime}=\left[\begin{array}{lllll}
z(1) & z(2) & \ldots & z(N)
\end{array}\right], \\
\mathbf{v}^{\prime}{ }_{N}=\left[\begin{array}{lllll}
v(1) & v(2) & \ldots & v(N)
\end{array}\right], & \mathbf{e}_{N}^{\prime}=\left[\begin{array}{llll}
e(1) & e(2) & \ldots & e(N)
\end{array}\right], \\
\mathbf{G}=\sum_{i=0}^{\infty} g_{i} \mathbf{S}^{i}, & \mathbf{H}=\sum_{i=0}^{\infty} h_{i} \mathbf{S}^{i},
\end{aligned}
$$

$v(t)$ ие $(t)$ - процессы белого шума с нулевыми средними. Предположим, что $\sum_{i=0}^{\infty} g_{i}^{2}<\infty, \sum_{i=0}^{\infty} h_{i}^{2}<\infty u v(t)=e(t)$ или процессы $v(t), e(t)$ взаимно независимы. Tozda

$$
\lim _{N \rightarrow \infty} \frac{1}{N} \mathbf{z}_{N}^{\prime} \mathbf{x}_{N}=E[e(t) v(t)] \sum_{i=0}^{\infty} h_{i} g_{i}
$$

n. $H$.

Л ем а 2. Если $\mathbf{H}=\mathrm{KT}^{-1}$, где $\mathrm{K}$ и т нижние треугольные теплицевы матриць, $k_{i}=0, i>m$ и теплицева матрица Т отвечает полиному, корни которого лежат вне единичного круга, то

$$
\sum_{j=0}^{\infty} h_{j}^{2}<\infty
$$

Доказательство. Таккак $h_{j}=\sum_{i=0}^{m} k_{i} t_{j-i}$, то

$$
\begin{gathered}
\sum_{j=0}^{\infty} h_{j}^{2}=\sum_{i=0}^{m} \sum_{l=0}^{m} k_{i} k_{l} \sum_{j=0}^{\infty} t_{j-i} t_{j-l} \leqslant \sum_{i=0}^{m} \sum_{l=0}^{m} k_{i} k_{l}\left(\sum_{j=0}^{\infty} t_{j-i}^{2} \sum_{j=0}^{\infty} t_{j-l}^{2}\right)^{1 / 2} \leqslant \\
\leqslant \sum_{i=0}^{m} \sum_{l=0}^{m} k_{i} k_{l} \sum_{j=0}^{\infty}\left|t_{j-i}\right| \sum_{j=0}^{\infty}\left|t_{j-l}\right|<\infty .
\end{gathered}
$$

Первое неравенство следует из неравенства Коши-Буняковского, а последнее - в силу предположения леммы 2.

Л е м а 3. Если для системы (3), (4) справедливо условие А, то

$$
J^{a}(\theta) \triangleq \lim _{N \rightarrow \infty} \frac{1}{N} J_{N}^{a}(\theta)=E J_{N}^{a}(\theta)=1+\left(1+\theta^{\prime}{ }_{1} \theta_{1}\right)^{-1}\left[\sum_{i=0}^{\infty} h_{i}^{2}+2 \sum_{i=0}^{n_{a}} h_{i} a_{i}\right] .
$$


Д оказ ательство. Из (3), (4) имеем

$$
\begin{aligned}
& \mathbf{y}_{N}=\left(\mathbf{P}^{0}\right)^{-1} \mathbf{A}^{0} \mathbf{F} \varepsilon_{N}, \\
& \mathbf{u}_{N}=\left(\mathbf{P}^{0}\right)^{-1} \mathbf{A}^{0} \mathbf{D} \varepsilon_{N},
\end{aligned}
$$

следовательно,

$$
\begin{gathered}
\mathbf{A y}_{N}-\mathbf{B u}_{N}=\mathbf{A A}^{0} \mathbf{F}\left(\mathbf{P}^{0}\right)^{-1} \varepsilon_{N}-\mathbf{B D A}^{0}\left(\mathbf{P}^{0}\right)^{-1} \varepsilon_{N}= \\
=\mathbf{A} \varepsilon_{N}+\left(\mathbf{A B}^{0}-\mathbf{A}^{0} \mathbf{B}\right) \mathbf{D}\left(\mathbf{P}^{0}\right)^{-1} \varepsilon_{N} .
\end{gathered}
$$

Введя обозначение $\mathbf{H}=\left(\mathbf{A B}^{0}-\mathbf{A}^{0} \mathbf{B}\right) \mathbf{D}\left(\mathbf{P}^{0}\right)^{-1}$, находим

$$
\frac{1}{N} J_{N}^{a}(\theta)=\frac{1}{N \sigma_{\varepsilon}^{2}\left(1+\theta^{\prime}{ }_{1} \theta_{1}\right)}\left[\varepsilon^{\prime}{ }_{N} \mathbf{A}^{\prime} \mathbf{A} \varepsilon_{N}-1-\varepsilon^{\prime}{ }_{N} \mathbf{H}^{\prime} \mathbf{H} \varepsilon_{N}+2 \varepsilon^{\prime}{ }_{N} \mathbf{A}^{\prime} \mathbf{H} \varepsilon_{N}\right] \text {. }
$$

Так как по лемме $2 \sum_{i=0}^{\infty} h_{i}{ }^{2}<\infty$, то, используя лемму 1 , получим

$$
J^{a}(\theta)=1+\left(1+\theta^{\prime}{ }_{1} \theta_{1}\right)^{-1}\left[\sum_{i=0}^{\infty} h_{i}^{2}+2 \sum_{i=0}^{n_{a}} h_{i} a_{i}\right] .
$$

Л ем м а 4. Если для системь (3), (4) справедливы условия А, Б, В1, то при $\theta \neq \theta^{0}$

$$
J^{a}(\theta)>J^{a}\left(\theta^{0}\right)
$$

Д ок а з а те льст в о. Заметим, что $J^{a}\left(\theta^{0}\right) \downarrow 1$. Если справедливо условие В1, то при $i \leqslant n_{a}$ имеем $h_{i}=0$, а также $\sum_{i=0}^{n_{a}} h_{i} a_{i}=0 . \quad$ Следовательно,

$$
J^{a}(\theta)=1+\left(1+\theta^{\prime}{ }_{1} \theta_{1}\right)^{-1} \sum_{i=0}^{\infty} h_{i}^{2} \geqslant 1 .
$$

Равенство достигается тогда и только тогда, когда элементы $h_{i}$ теплицевой матрицы $\mathbf{H}$ при $i=0,1,2, \ldots$ равны нулю, т. е. когда полином $H(z)$, соответствующий матрице $\mathbf{H},-$ нулевой:

или

$$
H(z) \triangleq\left[A(z) B^{0}(z)-A^{0}(z) B(z)\right] D(z)\left[P^{0}(z)\right]^{-1}=0
$$

$$
\left[A(z)-A^{0}(z)\right] B^{0}(z)-\left[B(z)-B^{0}(z)\right] A^{0}(z)=0 .
$$

Это однородная система линейных уравнений относительно неизвестных $a_{i}-a_{i}{ }^{0}, i=1,2, \ldots, n_{a}$ и $b_{i}-b_{i}{ }^{0}, i=p, p+1, \ldots, n_{b}$. По условию Б в системе $n_{a}+n_{b}-p+1$ независимых уравнений, следовательно, она имеет только нулевое решение $a_{i}-a_{i}{ }^{0}=0, i=1,2, \ldots, n_{a}$, $b_{i}-b_{i}{ }^{0}=0, i=p, p+1, \ldots, n_{b}$, что и доказывает лемму.

Л е м м 5. Если в системе (3), (4) выполнены условия А, Б, В1, Д, то оценки собственного вектора параметров обтекта, минимизирующие $\frac{1}{N} J_{N}^{a}(\theta)$ на множестве $\Theta$, сходятся $п$. н. к истинному $\theta^{0}$ при $N \rightarrow \infty$. Доказ ательство. Пусть $\hat{\theta}_{N}$ - оценки собственного вектора, минимизирующие $\frac{1}{N} J_{N}^{a}(\theta), \quad \theta \in \Theta$. Следовательно, $J_{N}^{a \bullet}\left(\hat{\theta}_{N}\right) \leqslant J_{N}^{a}\left(\theta^{0}\right)$. По 
условию Д последовательность $\hat{\theta}_{N}$ имеет сходящуюся подпоследовательность $\hat{\theta}_{N_{n}}, \lim _{n \rightarrow \infty} \hat{\theta}_{N_{n}}=\theta^{*}$. Допустим, что $\theta^{*} \notin \mathrm{S}_{\varepsilon}$, где $S_{\varepsilon}=$ $=\left\{\theta\right.$ : inf $\left.\left\|\theta^{0}-\theta\right\|\right\}<\varepsilon$, и покажем, что это предположение противоречит условию, которому, по определению, должны удовлетворять оценки собственного вектора. Справедливо равенство

$$
\delta\left(\theta^{*}\right)=\left|J^{a}\left(\theta^{*}\right)-J^{a}\left(\theta^{0}\right)\right| .
$$

Так как $\frac{1}{N} J_{N}^{a}\left(\theta^{*}\right)$ и $\frac{1}{N} J_{N}^{a}\left(\theta^{0}\right)$ сходятся п. н. с ростом выборки к $J\left(\theta^{*}\right)$ и $J\left(\theta^{0}\right)$ соответственно, то на множестве $\Theta$ найдется $N_{1}$ такое, что при $N_{n}>N_{1}$

$$
\begin{aligned}
& \left|\frac{1}{N_{n}} J_{N_{n}}^{a}\left(\theta^{*}\right)-J^{a}\left(\theta^{*}\right)\right|<\frac{\delta}{4}, \\
& \left|\frac{1}{N_{n}} J_{N_{n}}^{a}\left(\theta^{0}\right)-J^{a}\left(\theta^{0}\right)\right|<\frac{\delta}{4}
\end{aligned}
$$

п. н. Так как подпоследовательность $\hat{\theta}_{N_{n}}$ сходится п. н. к. $\theta^{*}$ при $n \rightarrow \infty$, а функция $\frac{1}{N} J_{N}^{a}(\theta)$ непрерывна по $\theta$, то найдется $N_{2}$ такое, что при $N_{n}>N_{2}$ выполняется п. н.

$$
\left|\frac{1}{N_{n}} J_{N_{n}}^{a}\left(\hat{\theta}_{N_{n}}\right)-\frac{1}{N_{n}} J_{N_{n}}^{a}\left(\theta^{*}\right)\right|<\frac{\delta}{4} .
$$

Следовательно, найдется $N_{0} \geqslant \max \left(N_{1}, N_{2}\right)$ такое, что для $N_{n} \geqslant N_{0}$

$$
\begin{aligned}
& \left|\frac{1}{N_{n}} J_{N_{n}}^{a}\left(\hat{\theta}_{N_{n}}\right)-J^{a}\left(\theta^{*}\right)\right|<\frac{\delta}{2}, \\
& \left|\frac{1}{N_{n}} J_{N_{n}}^{a}\left(\theta^{0}\right)-J^{a}\left(\theta^{0}\right)\right|<\frac{\delta}{2}
\end{aligned}
$$

п. н. Из (7) следует, что при $N_{n} \geqslant N_{0}$

$$
\begin{aligned}
\mid J^{a}\left(\theta^{*}\right)-J^{a}\left(\theta^{0}\right) & +\frac{1}{N_{n}} J_{N_{n}}^{a}\left(\theta^{0}\right)-\frac{1}{N_{n}} J_{N_{n}}^{a}\left(\hat{\theta}_{N_{n}}\right) \mid<\delta= \\
& =\left|J^{a}\left(\theta^{*}\right)-J^{a}\left(\theta^{0}\right)\right|,
\end{aligned}
$$

откуда

$$
J_{N_{n}}^{a}\left(\hat{\theta}_{N_{n}}\right)>J_{N_{n}}^{a}\left(\theta^{0}\right) .
$$

Поскольку $\varepsilon$ произволен, то полученное противоречие доказывает лемму.

\section{5. Результаты эксперимента на ЦВМ}

С целью иллюстрации выводов предыдущего пункта исследование метода собственного вектора при предположениях теоремы 1 проводилось на трех моделях объектов. 
1. Модель колебательного объекта $\left[{ }^{12}\right]$

$$
\begin{gathered}
y(t)-1,5 y(t-1)+0,7 y(t-2)= \\
=u(t-1)+0,5 u(t-2)+\varepsilon(t)-1,5 \varepsilon(t-1)+0,7 \varepsilon(t-2)
\end{gathered}
$$

и обратная связь

$$
u(t)=0,1\left[y^{*}-y(t-2)\right] .
$$

2. Модель неминимальнофазового объекта $\left[{ }^{12}\right]$

$$
\begin{gathered}
y(t)-1,43 y(t-1)+0,47 y(t-2)= \\
=-0,10 u(t-1)+0,17 u(t-2)+\varepsilon(t)-1,43 \varepsilon(t-1)+0,47 \varepsilon(t-2)
\end{gathered}
$$

и обратная связь

$$
u(t)=1,5\left[y^{*}-y(t-2)\right] .
$$

3. Модель третьего объекта получена из первого в предположении, что $b_{1}=0$, т. е.

$$
\begin{gathered}
y(t)-1,5 y(t-1)+0,7 y(t-2)= \\
=0,5 u(t-2)+\varepsilon(t)-1,5 \varepsilon(t-1)+0,7 \varepsilon(t-2),
\end{gathered}
$$

и обратная связь

$$
u(t)=-0,1 u(t-2)+0,25\left[y^{*}-y(t-1)\right] .
$$

\begin{tabular}{|c|c|c|c|c|c|c|}
\hline & \multicolumn{2}{|c|}{ Модель I } & \multicolumn{2}{|c|}{ Модель II } & \multicolumn{2}{|c|}{ Модель III } \\
\hline & $\begin{array}{c}\text { Истин- } \\
\text { ное } \\
\text { значе- } \\
\text { ние }\end{array}$ & Оценка & $\begin{array}{c}\text { Истин- } \\
\text { ное } \\
\text { значе- } \\
\text { ние }\end{array}$ & Чценка & $\begin{array}{c}\text { Истин- } \\
\text { ное } \\
\text { значе- } \\
\text { ние }\end{array}$ & Оценка \\
\hline $\begin{array}{c}2,5 \\
25 \\
250\end{array}$ & $-1,5$ & $\begin{array}{l}-1,50 \\
-1,45 \\
-1,50\end{array}$ & $-1,43$ & $\begin{array}{l}-1,64 \\
-1,42 \\
-1,42\end{array}$ & $-1,5$ & $\begin{array}{l}-1,71 \\
-1,56 \\
-1,50\end{array}$ \\
\hline $\begin{array}{c}2,5 \\
25 \\
250\end{array}$ & 0,7 & $\begin{array}{l}0,73 \\
0,67 \\
0,70\end{array}$ & 0,47 & $\begin{array}{l}0,69 \\
0,46 \\
0,46\end{array}$ & 0,7 & $\begin{array}{l}0,89 \\
0,74 \\
0,70\end{array}$ \\
\hline $\begin{array}{r}2,5 \\
25 \\
250\end{array}$ & 1 & $\begin{array}{l}0,33 \\
0,52 \\
0,97\end{array}$ & $-0,10$ & $\begin{array}{l}-0,17 \\
-0,13 \\
-0,10\end{array}$ & 0,5 & $\begin{array}{l}0,32 \\
0,46 \\
0,50\end{array}$ \\
\hline $\begin{array}{c}2,5 \\
25 \\
250\end{array}$ & 0,5 & $\begin{array}{l}1.11 \\
1.13 \\
0,54\end{array}$ & 0,17 & $\begin{array}{l}0,23 \\
0,20 \\
0,17\end{array}$ & E & $=$ \\
\hline
\end{tabular}

Реализации процессов $y(t), u(t) \quad(N=500)$ генерировались из последовательностей дискретного белого шума согласно уравнениям (8) и (8a), (9) и (9a), (10) и (10a) соответственно при различных величинах $\eta=y^{*} / \sigma_{\varepsilon}$, где $y^{*}$ - задающее воздействие регулятора. По реализациям вычислялись оценки собственного вектора параметров. Результаты эксперимента приведены в таблице.

Результаты статистического моделирования 
ЛИТЕРА Т У РА

1. Gustavsson, I., Ljung, L., Söderström, T., Automatica, 13, № 1, 5975 (1977).

2. Wellste ad, P. E., Edmunds, J. M., Int. J. Contr., 21, № 4, 689-699 (1975).

3. L jung, L., IEEE Trans. Automat. Contr., 21, № 5, 779-781 (1976).

4. В орч и к Б. Г., Автоматика и телемеханика, № 4, 32-48 (1975).

5. В о р ч и к Б. Г., Автоматика и телемеханика, № 12, 32-48 (1976).

6. Söderström, T., Gustavsson, I., Ljung, L., Int. J. Contr., 21, № 2, 243-255 (1975).

7. Ljung, L., Gustavsson, I., Söderström, T., IEEE Trans. Automat. Contr., 19, № 6, 836-840 (1974).

8. A o ki, M., Yue, P. C., IEEE Trans. Automat. Contr., 15, № 5, 541-548 (1970).

9. Åström, K. J., B ohli n, T., Wensmark, S., Automatic construction of linear dynamic models for stationary industrial processes with random disturbances using operating records, Techn. Paper 18.150, IBM Nordic Lab., Sweden, June 1, 1965.

10. З а де Л., Д е з о е р Ч., Теория линейных систем, М., «Наука», 1970.

11. Söderström, T., Problems of Control and Information Theory, 4, № 2, 131138 (1975)

12. I s e rm a nn, R., B a ur, U., B a mberger, W., Kneppo, P., Siebert, A., Automatica, 10, № 1, 81-103 (1974).

Институт кибернетики

Академии наук Эстонской ССР
Поступила в редакцию

$10 / \mathrm{V} 1978$

\section{Olle KOTTA}

\section{SULETUD AHELAS TOIMUVATE LINEAARSETE PROTSESSIDE IDENTIFITSEERIMINE}

Artiklis on vaadeldud suletud süsteemide identifitseerimist omavektormeetodil ja maksimaalse tõepärasuse meetodil. Identifitseeritav objekt on lineaarne diskreetne statsionaarne ühe sisendi ja ühe väljundiga süsteem, mida juhitakse tagasiside abil lineaarse statsionaarse regulaatoriga. On saadud piisavad tingimused objekti parameetrite omavektorhinnangute ja maksimaalse tōepärasuse hinnangute tugevaks konsistentsuseks. Tulemusi on illustreeritud mõne simuleerimiseksperimendiga.

\section{Olle KOTTA}

\section{IDENTIFICATION OF LINEAR PROCESSES IN CLOSED LOOP}

Despite the importance of closed-loop identification, most theoretical results on the behavior of identification methods are not valid for systems operating in closed loop. It is therefore of vital importance to know under what conditions it is actually possible to obtain reliable identitication results for systems operating under closed-loop conditions. The result of an identification experiment depends clearly on several items: the system, the model structure, the identification method used, the experimental conditions. In this paper two different identification methods - the eigenvector method and the maximum likelihood method are discussed. It is assumed that the system is linear stationary single input- single output and that it is given as a difference equation in the input and output signals. The feedback is assumed to be linear, time-invariant and noise free. Results are obtained which determine sufficient conditions for strong consistency of considered estimates of system parameters. In particular, it is shown that the eigenvector estimates are consistent if there is at least $n+1$ delays in the loop (where by $n$ is denoted the system order) and maximum likelihood estimates are consistent if there is at least one delay in the loop (either in the system or in the regulator). The results are illustrated by a few simulation experiments. 